\title{
RESULTS OF THE FIRST SIMULTANEOUS X-RAY, OPTICAL, AND RADIO CAMPAIGN ON THE BLAZAR
} PKS 1622-297

\author{
Angela Osterman Meyer ${ }^{1,2}$, H. Richard Miller ${ }^{2}$, Kevin Marshall $^{3}$, Wesley T. Ryle $^{2}$, Hugh Aller $^{4}$, Margo Aller $^{4}$, \\ John P. McFarland ${ }^{5}$, Joseph T. Pollock ${ }^{6}$, Daniel E. Reichart ${ }^{7}$, J. Adam Crain ${ }^{7}$, Kevin M. IVARsen $^{7}$, \\ Aaron P. LaCluyze ${ }^{7}$, and Melissa C. NySewander ${ }^{7,8}$ \\ ${ }^{1}$ Egan Observatory, College of Arts and Sciences, Florida Gulf Coast University, 10501 FGCU Blvd, S., Fort Myers, FL 33965, USA; ameyer@ fgcu.edu \\ ${ }_{2}^{2}$ Department of Physics and Astronomy, Georgia State University, 1 Park Place ste. 730, Atlanta, GA 30303, USA \\ ${ }^{3}$ Department of Physics and Astronomy, Bucknell University, 701 Moore Avenue, Lewisburg, PA 17837, USA \\ ${ }^{4}$ Department of Astronomy, University of Michigan, 500 Church St. 830 Dennison, Ann Arbor, MI 48109-1042, USA \\ ${ }^{5}$ Kapteyn Instituut, Rijksuniversiteit Groningen, 9747 AD Groningen, The Netherlands \\ ${ }^{6}$ Department of Physics and Astronomy, Appalachian State University, 231 CAP Building, 525 Rivers Street, Boone, NC 28608, USA \\ ${ }^{7}$ Department of Physics and Astronomy, University of North Carolina at Chapel Hill, CB 3255, Phillips Hall, Chapel Hill, NC 27599-3255, USA \\ ${ }^{8}$ Space Telescope Science Institute, 3700 San Martin Drive, Baltimore, MD 21218, USA \\ Received 2007 August 7; accepted 2008 July 14; published 2008 August 15
}

\begin{abstract}
Coordinated X-ray, optical, and radio observations of the blazar PKS 1622-297 were obtained during a three-week campaign in 2006 using the Rossi X-Ray Timing Explorer (RXTE), the University of Michigan Radio Astronomy Observatory, and optical telescopes at Cerro Tololo Inter-American Observatory. The $R X T E$ observations indicate that this object is a comparatively weak X-ray emitter for a Flat-Spectrum Radio Quasar. The observed broadband spectral shape indicates that X-rays were most likely produced by the Inverse Compton processes. Optical observations of this object produced unexpected results in that this object appeared redder when in a bright state and bluer when in a faint state, contrary to the observed behavior of BL Lac objects.
\end{abstract}

Key words: galaxies: active - galaxies: individual (PKS 1622-297)

\section{INTRODUCTION}

Blazars are a radio-loud subclass of active galactic nuclei (AGNs), typically featuring core-dominated radio morphologies. Their optical continua are featureless and markedly steep; they exhibit a high degree of polarization (up to about 20\%), and their fluxes vary on timescales of hours to years at all wavelengths. The most extreme and unique property of blazars is their highly beamed continuum, most likely produced by a jet of relativistic material oriented close to the observer's line of sight (see Urry \& Padovani 1995, and references therein for further details).

Blazars may be subdivided into two categories: BL Lac objects and Flat-Spectrum Radio Quasars (FSRQs). BL Lac objects have particularly weak spectral lines, so their redshifts, and hence luminosities, are difficult to determine. FSRQs are distinguished by the presence of broad emission lines in their optical spectra. The distinction between the two classes may become blurred as a result of their variability. Spectral lines indicating an FSRQ may be present during a low continuum state, but such lines may become weak or disappear during a high continuum state, thus resembling a BL Lac object (see Ulrich et al. 1997, and references therein for further details).

The spectral energy distribution (SED) of blazars is distinguished by two peaks: one in the radio/UV regime and the other in the X-ray $/ \gamma$-ray regime. The spectrum in the radio-toUV range is generally agreed to arise from synchrotron emission from relativistic electrons spiraling around the jet's magnetic field lines (Ulrich et al. 1997). For low-frequency-peaked BL Lac objects (LBLs), the X-ray/ $\gamma$-ray spectrum is most likely due to inverse Compton (IC) emission. However, in high-frequency-peaked BL Lac objects (HBLs), the X-rays are thought to be the high-energy tail of the synchrotron emission (Urry \& Padovani 1995). In order to fully understand blazars, we must understand the physics of the region near the central engine, since this is where jet particles are collimated and accelerated to relativistic speeds (Ulrich et al. 1997).

PKS 1622-297 has not been well studied, in large part due to its faintness and its location close to the galactic center. This object was first detected in the Parkes Catalog (Shimmins \& Bolton 1974). Radio observations and spectral studies by Kuehr et al. (1981) indicated that it is a blazar. The optical counterpart of PKS 1622-297 was identified by Torres \& Wroblewski (1984). Wright \& Otrupcek (1990) report a redshift of $z=$ 0.815. Its SED (Mattox \& Wagner 1996) identifies it as an FSRQ. Subsequent results from Ghisellini et al. (1998) also classify this object as a low-polorization quasar (LPQ). It was observed several times with EGRET (Hartman et al. 1999), which detected a large high-energy flare in 1995 June. This flare was the largest EGRET flare observed for any blazar, with a photon flux of $21 \pm 7 \times 10^{-6} \mathrm{~cm}^{-2} \mathrm{~s}^{-1}$ at $E>100 \mathrm{MeV}$ (Mattox $\&$ Wagner 1996). Few multiwavelength campaigns have been performed on PKS 1622-297.

In this work, we present the results of the first simultaneous X-ray, optical, and radio campaign on PKS 1622-297. In Section 2, we summarize the observational results and provide details of the observational and data reduction procedures. In Section 3, we discuss the surprising color variations observed at optical wavelengths. We perform broadband spectral analysis in Section 4 before summarizing our conclusions in Section 5.

\section{OBSERVATIONS AND DATA REDUCTION}

PKS 1622-297 has been monitored optically for more than a decade by the Program for Extragalactic Astronomy (PEGA) at Georgia State University (GSU; see Figure 1). Observations of PKS 1622-297 during 2006 April-May revealed the optical brightness steadily increasing. As a result of this optical outburst, a Rossi X-Ray Timing Explorer (RXTE) Target of Opportunity (ToO) for this object was triggered (when the 


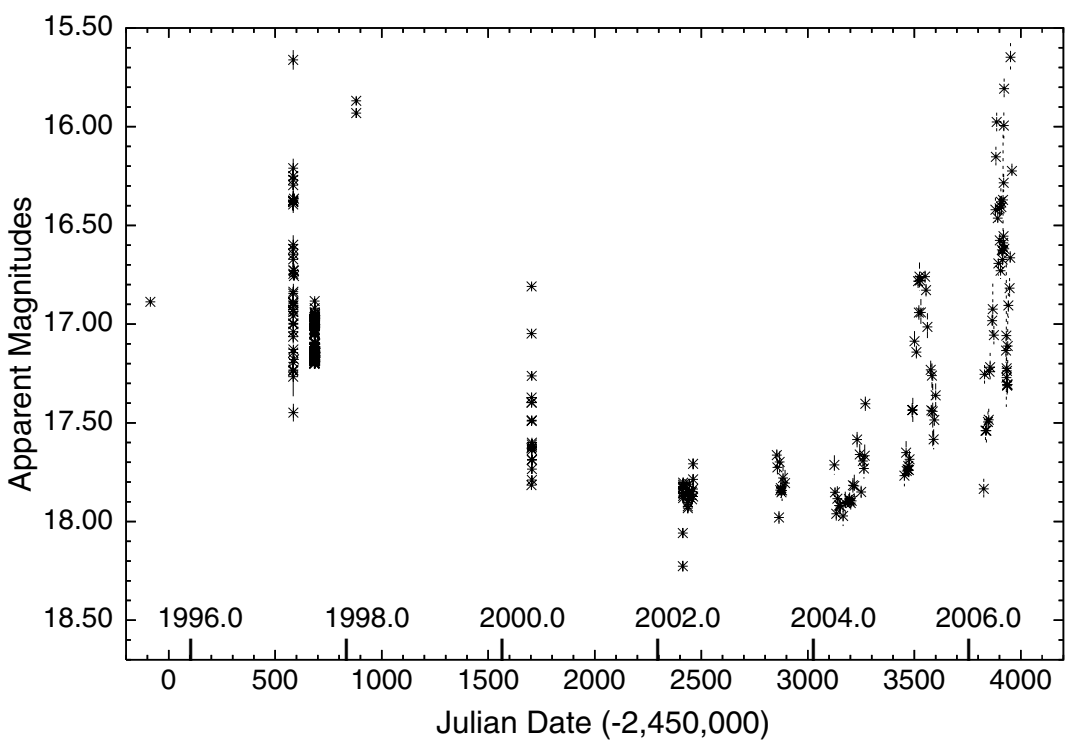

Figure 1. Long-term $R$-band activity observed in PKS 1622-297.

Table 1

Summary of Observations

\begin{tabular}{lccc}
\hline \hline Observatory & Spectral Band & Dates of Observations & No of Observations \\
\hline$R X T E$ & 2-10 keV & 2006 May 31, Jun 27-Jul 26 & 46 \\
PROMPT2 & $B$-band & 2006 Jun 29-Jul 15 & 4 \\
PROMPT4 & $V$-band & 2006 Jun 29-Jul 21 & 12 \\
& $R$-band & 2006 Jun 29-Jul 21 & 34 \\
& $I$-band & 2006 Jun 29-Jul 21 & 32 \\
SMARTS & $B$-band & 2006 Mar 31-Aug 10 & 43 \\
& $V$-band & 2006 Mar 31-Aug 10 & 44 \\
& $R$-band & 2006 Mar 31-Aug 10 & 45 \\
CTIO 1.0m & $R$-band & 2006 Jun 13 & 61 \\
UMRAO & $14.5 \mathrm{GHz}$ & 2006 May 31-Aug 4 & 7 \\
& $8.0 \mathrm{GHz}$ & 2006 May 29-Jul 13 & 6 \\
& $4.8 \mathrm{GHz}$ & 2006 Jun 4-Jul 29 & 3 \\
\hline
\end{tabular}

object reached $R>16.5$, a high state for this usually dim object; see Figure 1). Coordinated multifrequency radio observations at the University of Michigan Radio Astronomy Observatory (UMRAO) were initiated near the same time. In mid-June, intensive $R$-band observations were obtained using the Yale $1.0 \mathrm{~m}$ telescope at the Cerro Tololo Inter-American Observatory (CTIO). Radio and optical observations of this object were obtained regularly throughout June. The full RXTE campaign on PKS 1622-297 was initiated at the end of June, lasting from June 27 to July 23. During this time, regular observations continued from UMRAO and were also conducted with the Small and Moderate Aperture Research Telescope System (SMARTS) $1.3 \mathrm{~m}$ telescopes. Optical BVRI observations were also obtained from the Panchromatic Robotic Optical Monitoring and Polarimetry Telescopes (PROMPT) P2 and P4 at CTIO. These data include intensive $R$ - and $I$-band observations on July 5 . The faintness of the object, particularly in the $B$ - and $V$-bands, prohibited reliable, intensive observations on the remaining nights. Consequently, only nightly averages were used for the majority of the PROMPT data. A summary of all 2006 PKS 1622-297 observations is given in Table 1.

\subsection{X-Ray Data Reduction}

The RXTE X-ray light curve was extracted using the FTOOLS v5.2 software package. During nearly all of our observations, Proportional Counter Units (PCUs) 1, 3, and 4 were turned off.
Therefore, data were extracted only from PCUs 0 and 2. Despite the loss of the propane layer on board PCU 0 during 2000 May, the signal-to-noise ratio $(\mathrm{S} / \mathrm{N})$ was much greater when using data from both PCUs 0 and 2. To further enhance the $\mathrm{S} / \mathrm{N}$, only data from layer 1 of the Proportional Counter Array (PCA) were analyzed. No data from the High-Energy X-Ray Timing Experiment (HEXTE) cluster or the other PCUs were used. All the data analyzed here were taken while the spacecraft was in STANDARD-2 data mode.

Data were extracted only when the target's Earth elevation angle was $>10 \mathrm{deg}$, pointing offset $<0.02 \mathrm{deg}$, PCUs 0 and 2 both on, the spacecraft more than $30 \mathrm{~min}$ after South Atlantic Anomaly (SAA) passage, and electron noise less than 0.1 units. Since the background response of the PCU is not well defined above $20 \mathrm{keV}$, only channels $0-44(2-20 \mathrm{keV})$ are included in this analysis.

Because the PCA is a non-imaging detector, background issues can be critically important during analysis. The faintmode "L7" model, developed by the PCA team, was used here. This model provides adequate background estimation for objects with less than $40 \mathrm{cnts} \mathrm{s}^{-1}$. Background files were extracted using pcabackest v3.0.

\subsection{Optical Data Reduction}

All of the observations were taken, reduced, and/or processed by the PEGA group at Georgia State University. Bias/zero and 


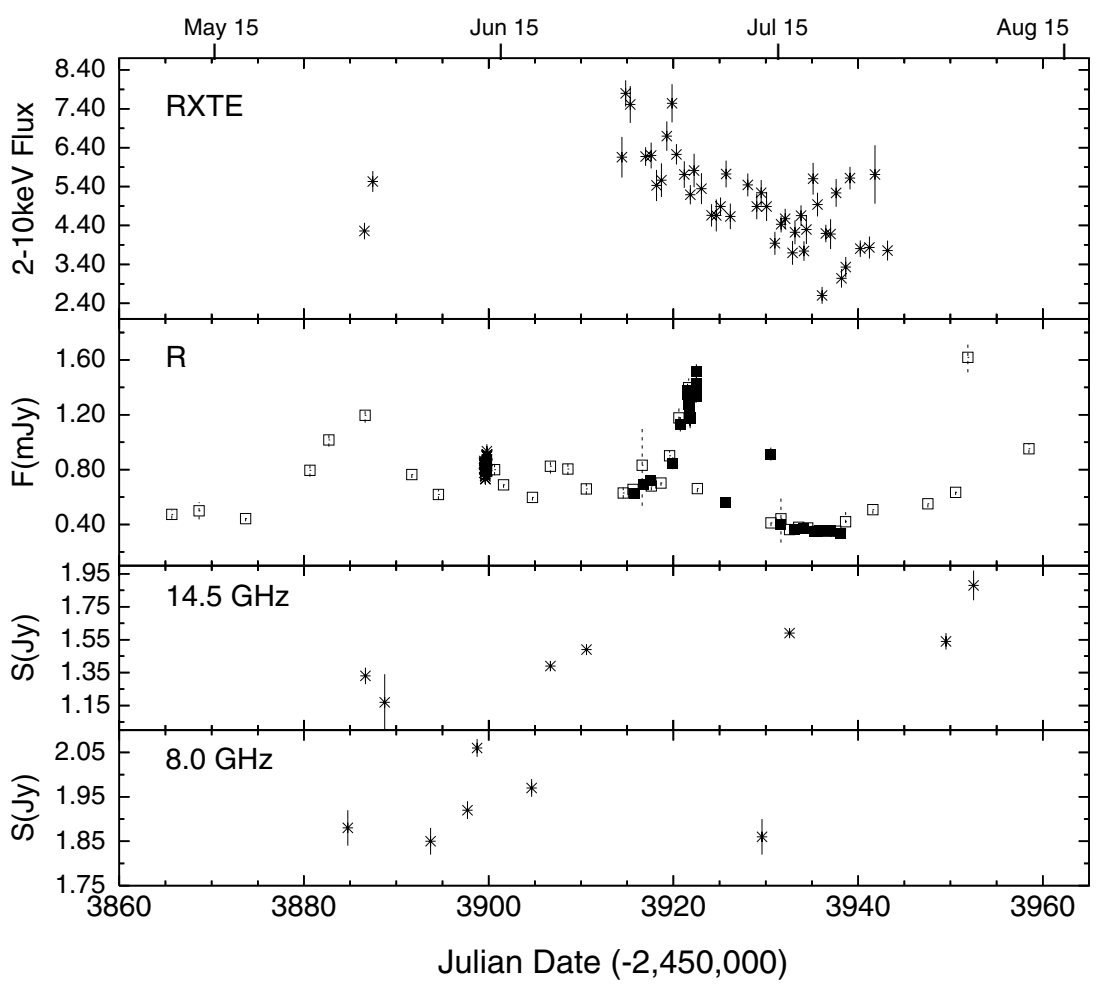

Figure 2. X-ray, optical, and radio activity observed during the 2006 multiwavelength campaign on PKS $1622-297$. The RXTE fluxes are given in units of $10^{-12}$ erg $\mathrm{cm}^{-2} \mathrm{~s}^{-1}$. For the optical data, the open squares indicate SMARTS data, the filled squares indicate PROMPT4 data, and the asterisks indicate CTIO data.

flat calibration frames were taken along with the PKS 1622-297 object frames. Dark calibration frames were not required for the Yale $1.0 \mathrm{~m}$ telescope's CCD since the chip contains a dark pixel strip. The object frames also included a number of comparison stars used to calibrate the observed brightness of PKS 1622-297 using differential photometry. The apparent magnitudes of the comparison stars for $B$-filtered frames are presented in Monet et al. (1998). The magnitude information of the comparison stars in $V$-, $R$-, and $I$-filtered frames is presented in González-Pérez et al. (2001). All data reduction utilized standard NOAO IRAF ${ }^{9}$ routines including ccdproc, flatcombine, and zerocombine. All data processing and 7 arcsec aperture photometry were performed using the ccdphot routine, written by Marc Buie, in IDL.

Many regularly spaced, longer-term observations were obtained from the SMARTS program's $1.3 \mathrm{~m}$ telescope. This telescope is equipped with a Fairchild 447 camera and Johnson color BVRI filters. The 2006 PKS 1622-297 campaign also utilized data from the Yale $1.0 \mathrm{~m}$ telescope and the PROMPT system, both located at CTIO. The Yale $1.0 \mathrm{~m}$ telescope is equipped with the Y4KCam STA CCD camera and uses Johnson $B V$ and Cousins $R_{c} I_{c}$ filters. Frames taken on this CCD were binned in order to reduce the readout time from almost a minute to only a few seconds. Observations from the Yale $1.0 \mathrm{~m}$ telescope were reduced and processed by the PEGA group. The PROMPT system currently uses five identical $0.41 \mathrm{~m}$ RitcheyChrétien telescopes equipped with fast readout Apogee Alta U47+ CCD cameras and Johnson BVRI filters. All raw images and calibration images from these telescopes were electronically transmitted to PEGA facilities for reduction and photometry.

9 IRAF is distributed by the National Optical Astronomy Observatories, which are operated by the Association of the Universities for Research in Astronomy, Inc., under cooperative agreement with the National Science Foundation.

\subsection{Radio Data Reduction}

The University of Michigan radio data were obtained using a $26 \mathrm{~m}$ prime focus paraboloid equipped with transistor-based radiometers operating at central frequencies of $4.8,8.0$, and $14.5 \mathrm{GHz}$ and room-temperature wide-band High Electron Mobility Pseudomorphic Transistor (HEMPT) amplifiers (with a width of $\sim 10 \%$ of the observing frequency). Measurements at all three frequencies utilized rotating, dual-horn polarimeter feed systems that permitted both total flux density and linear polarization to be measured. An on-off observing technique was used at $4.8 \mathrm{GHz}$, and an on-on technique at the other two frequencies. A typical observation consisted of 8-16 individual measurements over a $25-45$ min period (depending on frequency). A source selected from a grid of calibrators was observed for every $1-2 \mathrm{~h}$. The flux scale was set by observations of Cassiopeia A. Details of the calibration and analysis technique are given in Aller et al. (1985).

\subsection{Discussion of Observations}

The multiwavelength observations of PKS 1622-297, shown in Figure 2, display significant flaring activities at optical and $\mathrm{X}$-ray wavelengths. The June and July RXTE observations appeared to capture the peak and decline of a major flare, but the $\sim$ three week interruption in X-ray monitoring prevents one from precisely characterizing the nature of the variations. The optical data show two well-defined flaring events. The first occurs at the end of May, the second in early July. A third, sparsely sampled, sharp increase in flux occurs in early August. All of these events are present and correlated in the $B-, V-$, and $R$-bands. The flares occur simultaneously in these bands to within the time resolution of the observations. The sampling of the radio data is much sparser than that in the optical and Xray bands, but still yields interesting results. The $14.5 \mathrm{GHz}$ data 


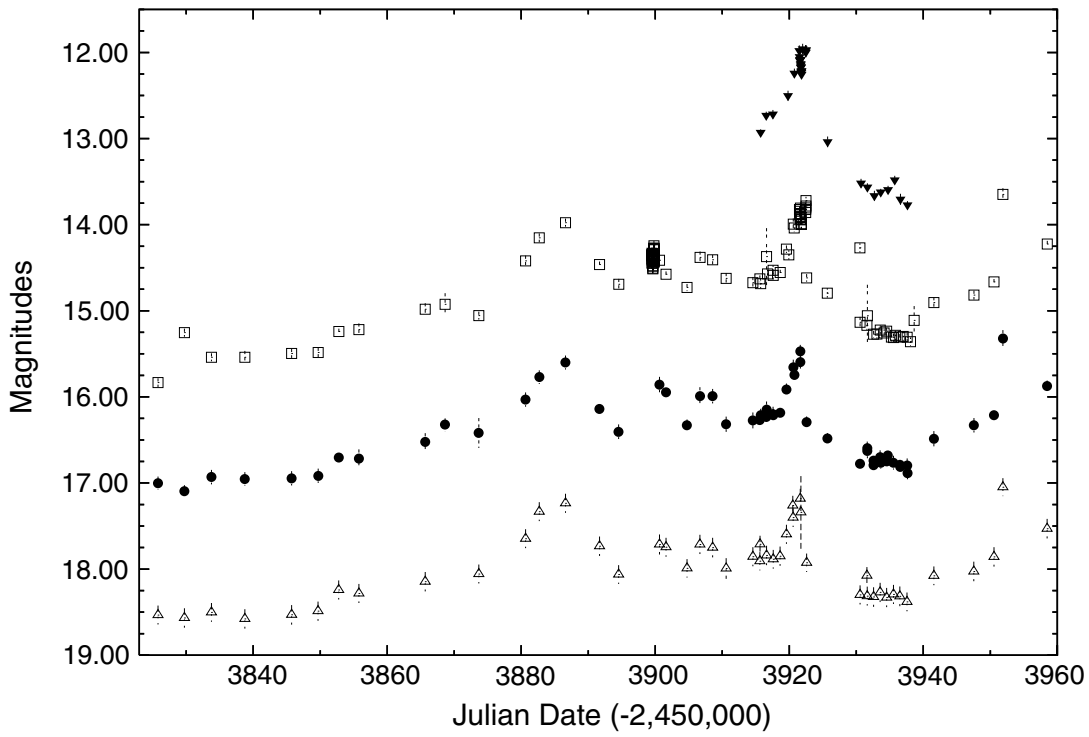

Figure 3. Observed optical brightness of PKS 1622-297 during the 2006 optical campaign. The filled inverted triangles represent $I$-band data, the open squares represent $R$-band data, the filled circles represent $V$-band data, and the open triangles represent $B$-band data. The $B$-band data are plotted at the correct scale, the $V$-band data have been offset by -1 magnitudes, the $R$-band data have been offset by -2 magnitudes, and the $I$-band data have been offset by -3 magnitudes.

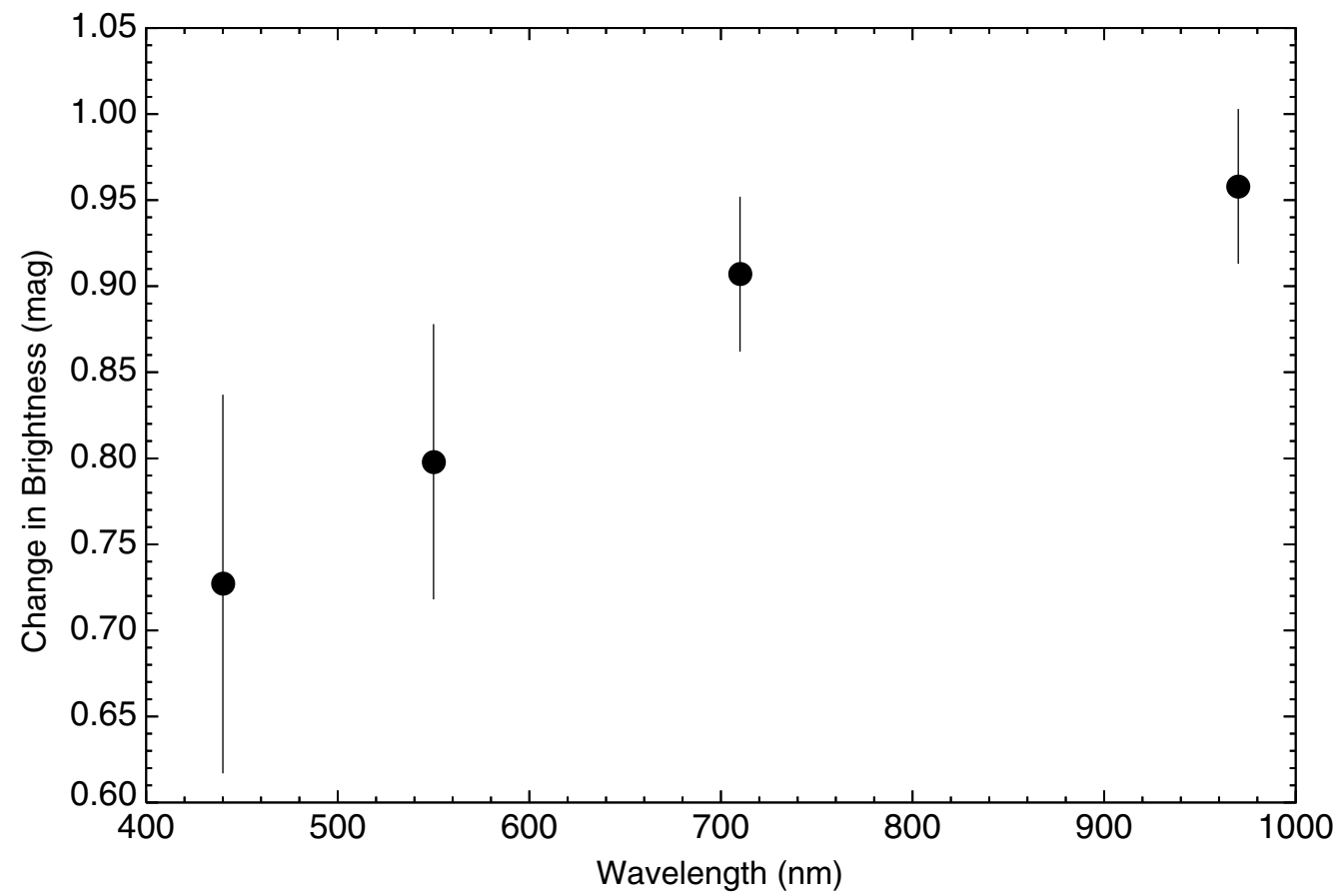

Figure 4. Illustration of the color change observed in PKS 1622-297 during a 2006 optical flux increase.

show a steady overall increase in flux throughout the summer. The $8.0 \mathrm{GHz}$ data show a sharp increase in flux of about $0.2 \mathrm{Jy}$ $(\sim 10 \sigma)$ over five days in early June. The irregular sampling of the radio data makes it difficult to determine whether or not the 14.5 and $8.0 \mathrm{GHz}$ radio activities are related to each other or to activity in the other observed wavebands.

During the 2006 campaign, many optical and X-ray flaring events were observed. The X-ray flare, assuming the peak was observed in late June and assuming the flare is symmetrical, lasted about 40 days. The flux peaked around July 1 and changes by a factor of 2 from peak to end. There were three prominent optical flaring events, each of which featured increases in flux by a factor of 2 to 3 in the $B-, V-, R$-, and $I$-bands. The flux increase of the second flare matches best with that of the
$\mathrm{X}$-ray flare. However, this flare was more rapid than the Xray flare, lasting only about 10 days. The earlier optical flare was the broadest, lasting about 20 days, but this timescale was still shorter than that of the X-ray flare. The first optical flare peaked around May 31, about a month before the observed peak of the X-ray flare. The second optical flare peaked several weeks later, around July 6. The later increase in optical flux appears to be too steep and too rapid to be readily related to the $\mathrm{X}$-ray flare. The $2006 \mathrm{X}$-ray and optical flaring events observed in PKS 1622-297 do not appear to be correlated. Crosscorrelation analysis performed on the optical and X-ray data did not result in any significant correlations.

The radio observations are comparatively sparse during this time. There is no clear correlation between the behavior 

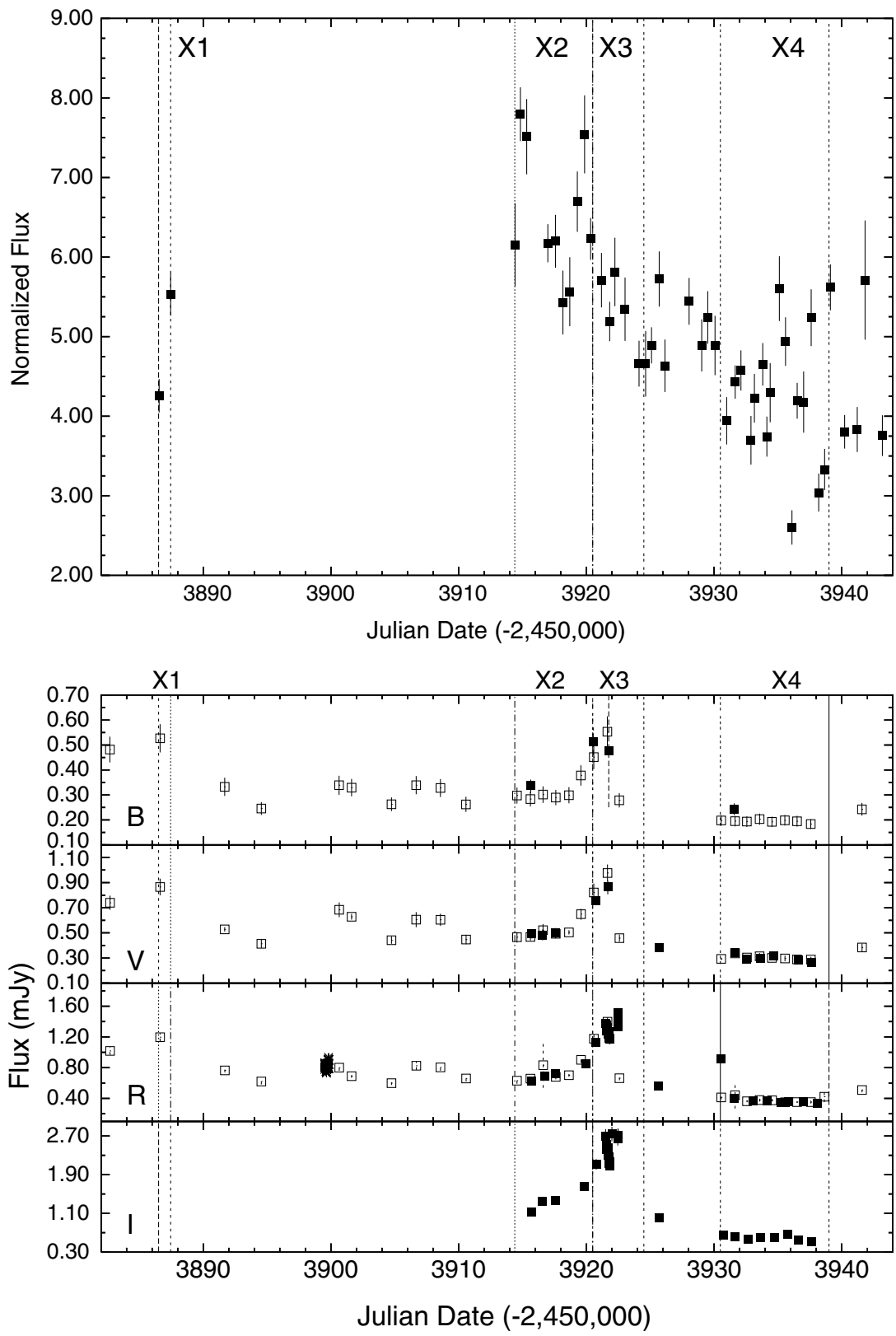

Figure 5. Simultaneous $R X T E$ and $R$-band observations, with labels $\mathrm{X} 1, \mathrm{X} 2, \mathrm{X} 3$, and $\mathrm{X} 4$ indicating time bins used for generating SEDs.

observed at radio wavelengths and that observed at optical wavelengths and X-ray wavelengths. However, the broad 14.5 GHz flux increase may be following and related to the increased level of activity observed at X-ray and optical wavelengths. This radio flux increase appears to lag the optical flare by $\sim 1$ month, but the lack of radio data around that time does not allow one to precisely determine the delay, if it is real.

\section{COLOR BEHAVIOR OBSERVED IN PKS 1622-297 AND SIMILAR OBJECTS}

Figure 3 displays only the optical brightness data from the 2006 PKS 1622-297 optical campaigns. The flaring structure is extremely similar across the observed wavebands. Figure 4 focuses on the flare observed at $I$ as well as $R, V$, and $B$ wavebands. During this flare, the observed change in brightness increases from shorter to longer wavelength bands. Contrary to what was expected based on previous observations of blazars, PKS 1622-297 became redder when brighter, not bluer. Similar analysis was performed on CTA 102, another FSRQ which was the subject of a 2005 simultaneous multiwavelength campaign (A. Osterman Meyer et al. 2008, in preparation). Again, the amplitude of each flare appears to increase from shorter to longer wavelength bands.

This surprising find in both CTA 102 and PKS 1622-297 lends support to the claims made on the few other occasions when this trend was observed. The first object reported to exhibit this effect was 3C 446 (Miller 1981). More recently, Ryle et al. (2007) found 3C 454.3 to be redder when flaring during its dramatic outburst in 2005. Gu et al. (2006) also observed 3C 454.3 to be redder when flaring, and also claimed to see this behavior in PKS 0420-01 and 3C 345, although the effect was much stronger in 3C 454.3. Archival observations of 3C 345 again show "redderwhen-brighter" behavior as displayed in Table 2. All of these 

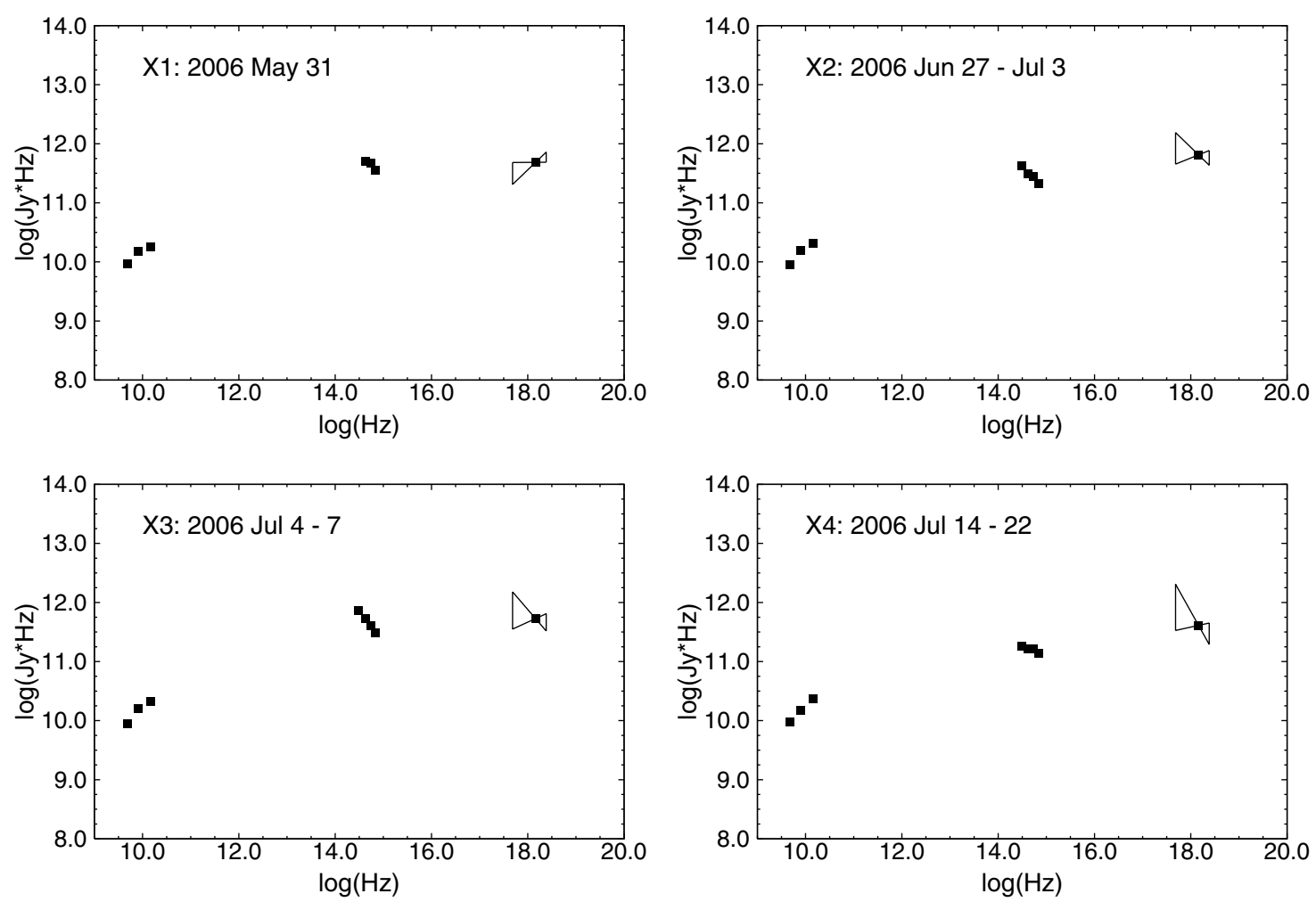

Figure 6. SEDs of PKS 1622-297 from the 2006 multiwavelength campaign.

Table 2

Archival 3C 345 Color Index Results

\begin{tabular}{lccr}
\hline \hline UT Date & $B^{\text {a }}$ & $V^{\text {a }}$ & $B-V$ \\
\hline 1971 Jul 18 & $15.51 \pm 0.18$ & $15.12 \pm 0.11$ & 0.39 \\
1971 Aug 27 & $15.50 \pm 0.27$ & $15.37 \pm 0.07$ & 0.13 \\
1971 Sep 22 & $15.47 \pm 0.10$ & $15.06 \pm 0.06$ & 0.41 \\
1971 Oct 09 & $15.42 \pm 0.08$ & $14.79 \pm 0.13$ & 0.63 \\
1971 Oct 11 & $15.33 \pm 0.09$ & $14.74 \pm 0.17$ & 0.59 \\
1971 Oct 12 & $15.34 \pm 0.04$ & $14.92 \pm 0.03$ & 0.42 \\
1972 May 21 & $15.98 \pm 0.05$ & $15.74 \pm 0.08$ & 0.24 \\
1972 Jun 03 & $16.26 \pm 0.04$ & $15.98 \pm 0.09$ & 0.28 \\
1972 Jun 04 & $16.15 \pm 0.02$ & $15.98 \pm 0.14$ & 0.17 \\
1972 Jun 09 & $16.58 \pm 0.15$ & $16.05 \pm 0.06$ & 0.53 \\
1972 Jun 12 & $16.20 \pm 0.14$ & $16.01 \pm 0.11$ & 0.19 \\
1972 Jun 14 & $16.23 \pm 0.09$ & $16.13 \pm 0.19$ & 0.10 \\
1972 Jul 03 & $16.43 \pm 0.11$ & $15.67 \pm 0.14$ & 0.76 \\
1972 Jul 09 & $15.93 \pm 0.13$ & $15.71 \pm 0.09$ & 0.22 \\
1973 Jul 24 & $16.72 \pm 0.12$ & $17.22 \pm 0.05$ & -0.50 \\
1973 Jul 26 & $16.83 \pm 0.17$ & $17.11 \pm 0.11$ & -0.28
\end{tabular}

Note. ${ }^{a}$ Data published in Webb et al. (1988).

objects are classified as FSRQs as well as Optically Violent Variables (OVVs).

Conversely, many low-frequency peaked BL Lac objects appear bluer when they are in a flaring state. BL Lac exhibited this trend during outbursts in 1997 (Nikolashvili \& Kurtanidze 2005) and 2001 (Villata et al. 2004; Stalin et al. 2006). Gu et al. (2006) also found BL Lac to be bluer when brighter, along with 3C 66A, PKS 0735+17, and OJ 287. Villata et al. (2004) observed BL Lac to be redder when in a fainter state, but observations by Benitez \& Ramirez (2006) found that BL Lac did not exhibit color changes when it was in a faint state.

$\mathrm{Gu}$ et al. (2006) propose that relative contributions of thermal versus nonthermal radiation could be responsible for the observed color behavior. Some FSRQs have been observed to contain a thermal "blue bump" component in their spectra, such as that observed for 3C 345 (Webb et al. 1994), possibly due to the accretion disk. In the scenario proposed by Gu et al. (2006), as an FSRQ brightens, the blue bump disappears and the optical continuum becomes redder. However, the blue bump component is sometimes small compared with the nonthermal synchrotron component even in low FSRQ state (see Webb et al. 1994). It may be that, in some states at least, the blue bump is not strong enough to cause significant color variations. Further UV observations of more objects are needed to fully understand the relationship between the blue bump and "redder-when-brighter" color behavior.

\section{BROADBAND SPECTRAL ANALYSIS}

To examine how the spectral shape, or SED, changed over time, the campaign on PKS 1622-297 was first split into four bins ranging from one day to a few days in size. The bins were defined based on times separating increases or decreases in the X-ray and optical flux. Figure 5 displays the RXTE data from each campaign with the bins, $\mathrm{X} 1-\mathrm{X} 4$, marked. This figure also displays the simultaneous optical data, again with bins $\mathrm{X} 1-\mathrm{X} 4$ labeled in time. The sampling of the radio data was not always dense enough at both frequencies $(8.0$ and $14.5 \mathrm{GHz})$ to provide simultaneous data for each time bin. However, the flux changes occur at slow enough rates at these frequencies so that nearly simultaneous averages of the few data taken during the RXTE campaign still accurately reflect the radio flux of each bin. The resulting SEDs for PKS 1622-297 are plotted in Figure 6 as the log of power in Jansky-Hertz versus the log of frequency in hertz. The optical differential magnitudes were converted to apparent magnitudes, and then to fluxes using the method of Allen (2001). An average frequency of $1.5 \times 10^{18} \mathrm{~Hz}$, corresponding to about $6 \mathrm{keV}$, was used for the RXTE data. The observed optical and radio spectra of PKS 1622-297 indicate 
that the synchrotron SED peak occurs somewhere between these two regimes. This agrees well with previous observations of similar objects.

The observed "redder-when-brighter" behavior has clear effects on the shape of the optical SED. The time bins labeled $\mathrm{X} 2$ and $\mathrm{X} 3$ in Figure 5 correspond to the beginning of and the peak of an optical flare, respectively. The bin X 4 corresponds to a low, steady flux level a few days after this flare. As displayed in Figure 6, the object's optical SED is steepest during the flare's peak in bin X3, with the SED sloping downward from the $I$-band to the $B$-band. The optical SED is less steep during the beginning of the flare in bin X2, and flattest during the low flux level of bin X4. The observed steepening of the optical SED again displays how PKS 1622-297 appears redder in a brighter flux state. A similar effect was observed by Miller (1981) and Sandage et al. (1966) in 3C 446. This observed optical steepening may agree with a model for optical variations proposed by Marscher (1980). In this model, flares in a blazar's synchrotron flux are produced by radiative thermal instabilities in the jet's relativistic flow. However, this model contains a number of parameters, so it is not feasible to firmly apply it to our observations of PKS 1622-297.

In objects such as PKS 1622-297, the X-ray emission is generally believed to be due to the IC process. Some observations of PKS 1622-297, and comparisons with observations of similar objects, suggest that this is the case. In the SEDs in Figure 6, for example, when compared with the X-ray powers and slopes, the optical powers and slopes indicate that the synchrotron portion of the spectrum cuts off below the X-ray regime, particularly in the X2 and X3 bins. Observations compiled from many simultaneous and quasi-simultaneous multiwavelength campaigns on 3C 279, also an FSRQ, compare best to models in which X-rays are produced by the synchrotron self-Compton process (SSC), where X-ray IC photons are produced from upscattered synchrotron photons (Hartman et al. 2001; Sikora et al. 2001).

During the EGRET era, an extremely large $\gamma$-ray flare was observed from PKS 1622-297 (Mattox \& Wagner 1996). Simultaneous X-ray measurements could not be obtained at the time of this flare. Such brightness in the $\gamma$-ray regime suggests that the X-ray emission is comparatively weak, at least at some times. Ghisellini et al. (1998) fit high-energy observations of PKS 1622-297 using both SSC and external radiation Compton (ERC) models, where the IC photons are produced from upscattered photons from a source external to the jet. The $\gamma$-ray emission fit well with both SSC and ERC models, but not many X-ray data were available for this investigation. Future observations of and computational models for this object would greatly benefit from concurrent X-ray and $\gamma$-ray observations.

\section{CONCLUSIONS}

In this work, we analyzed the results of the first coordinated radio, optical, and X-ray campaign on PKS 1622-297. The most exciting result is the discovery that PKS 1622-297 appears redder when in brighter optical flux states. Miller (1981) was the first to observe such behavior, in 3C 446. However, it was not until very recently that researchers found further examples of blazars that became redder, instead of bluer, during an optical flare ( $\mathrm{Gu}$ et al. 2006). This behavior may be due to the washing out of a UV thermal component in brighter FSRQ states. Future multiwavelength campaigns on this object should include UV observations to better investigate any thermal accretion disk component present in this object and how much this component may vary over time. Recent campaigns on similar objects have found significant blue bump contributions to the observed spectra (see for example, Kataoka et al. 2008). Additional optical multiwavelength campaigns on FSRQs as well as BL Lac objects are needed to fully determine why FSRQs frequently appear redder when brighter while BL Lacs are always observed to be bluer when brighter.

The X-ray emission in FSRQs is generally believed to be the result of the IC process. Our SEDs appear to support this conclusion. According to our 2006 observations, there appear to be no correlations between the optical flux activity and the less frequently sampled radio flux activity. Clements et al. (1995) find no significant correlations between long-term optical and radio behavior in FSRQs; it appears that the short-term activity also may not be correlated.

Future campaigns on blazars would benefit greatly from broader wavelength and longer temporal coverage. It would be very useful to include Gamma-Ray Large Area Space Telescope (GLAST) observations to investigate the high-energy behavior and possible correlations between $\gamma$-ray regime activity and activity observed at lower frequencies.

A.O.M., H.R.M., K.M., and W.T.R. are supported in part by PEGA's Research Program Enhancement funds from GSU. The RXTE observations were supported by NASA grants including NAG5-13733. A.O.M., H.R.M., K.M., and W.T.R. thank Lowell Observatory for generous allocations of observation time on the Perkins telescope with the Loral camera. A.O.M., H.R.M., and W.T.R. thank NOAO and CTIO for granting observation time and travel support for proposal 2006A-0044. The authors thank Todd Henry and the SMARTS consortium for contributing optical observations to this work. The UMRAO facility is partially supported by a series of grants from the NSF and by the University of Michigan. J.T.P., D.E.R., K.M.I., J.A.C., M.C.N., and A.P.L. thank the NSF's MRI and PREST programs, University of North Carolina at Chapel Hill, and Leonard Goodman for their support of the PROMPT observatory facility.

\section{REFERENCES}

Aller, H. D., Aller, M. F., Latimer, G. E., \& Hodge, P. E. 1985, ApJS, 59, 513 Allen, C. W., et al. 2001, Allen's Astrophysical Quantities (4th ed.; New York: Springer)

Benitez, E., \& Ramirez, A. 2006, in ASP Conf. Ser. 350, Blazar Variability Workshop II: Entering the GLAST Era, ed. H. R. Miller, K. Marshall, J. R. Webb, \& M. F. Aller (San Francisco, CA: ASP), 71

Clements, S. D., Smith, A. G., Aller, H. D., \& Aller, M. F. 1995, AJ, 110, 529

Ghisellini, G., Celotti, A., Fossati, G., Maraschi, L., \& Comastri, A. 1998, MNRAS, 301, 451

González-Pérez, J. N., Kidger, M. R., \& Martín-Luis, F. 2001, AJ, 122, 2055

Gu, M. F., Lee, C. U., Yim, H. S., \& Fletcher, A. B. 2006, A\&A, 450, 529

Hartman, R. C., et al. 1999, ApJS, 123, 79

Hartman, R. C., et al. 2001, ApJ, 553, 683

Kataoka, J., et al. 2008, ApJ, 672, 787

Kuehr, H., Witzel, A., Pauliny-Toth, I. I. K., \& Nauber, U. 1981, A\&AS, 45, 367

Marscher, A. P. 1980, ApJ, 239, 296

Mattox, J. R., \& Wagner, S. J. 1996, in ASP Conf. Ser. 110, Blazar Continuum Variability, ed. H. R. Miller, J. R. Webb, \& J. C. Noble (San Francisco, CA: ASP), 352

Miller, H. R. 1981, ApJ, 244, 426

Monet, D., et al. 1998, The USNO-A2.0 Catalogue (Washington, DC: US Naval Obs.)

Nikolashvili, M. G., \& Kurtanidze, O. M. 2005, Memorie della Societa Astronomica Italiana, 76, 55

Ryle, W. T., Miller, H. R., Marshall, K., Osterman, M. A., Carini, M. T., Boltwood, P., \& Aller, M. F. 2007, AAS Meeting Abstracts, 211, \#04.02

Sandage, A., Westphal, J. A., \& Strittmatter, P. A. 1966, ApJ, 146, 322

Shimmins, A. J., \& Bolton, J. G. 1974, Aust. J. Phys. Astrophys. Suppl., 32, 1 
Sikora, M., Błażejowski, M., Begelman, M. C., \& Moderski, R. 2001, ApJ, 554, 1

Stalin, C. S., Gopal-Krishna, , Sagar, R., Wiita, P. J., Mohan, V., \& Pandey, A. K. 2006, MNRAS, 366, 1337

Torres, C., \& Wroblewski, H. 1984, A\&A, 141, 271

Ulrich, M.-H., Maraschi, L., \& Urry, C. M. 1997, ARA\&A, 35, 445
Urry, C. M., \& Padovani, P. 1995, PASP, 107, 803

Villata, M., et al. 2004, A\&A, 421, 103

Webb, J. R., Smith, A. G., Leacock, R. J., Fitzgibbons, G. L., Gombola, P. P., \& Shepher, D. W. 1988, AJ, 95, 374

Webb, J. R., et al. 1994, ApJ, 422, 570

Wright, A. E., \& Otrupcek, R. 1990, in Parkes Radio Sources Catalog (Epping: ATNF) 